\begin{tabular}{|c|c|}
\hline Citation & $\begin{array}{l}\text { Hai Cao, Andrea Minoia, Inge De Cat, Johannes Seibel, Deepali Waghray, } \\
\text { Zhi Li, David Cornil, Kunal S. Mali, Roberto Lazzaroni, Wim Dehaen and } \\
\text { Steven De Feyter } \\
\text { Hierarchical self-assembly of enantiopure and racemic helicenes at the } \\
\text { liquid-solid interface from 2D to 3D } \\
\text { Nanoscale, 2017, 9, 18075-18080 }\end{array}$ \\
\hline Archived version & $\begin{array}{l}\text { Author manuscript: the content is identical to the content of the published } \\
\text { paper, but without the final typesetting by the publisher }\end{array}$ \\
\hline Published version & $\begin{array}{l}\text { insert link to the published version of your paper } \\
\text { http://dx.doi.org/10.1039/c7nr06700c }\end{array}$ \\
\hline Journal homepage & $\begin{array}{l}\text { insert link to the journal homepage of your paper http://www.rsc.org/journals- } \\
\text { books-databases/about-journals/nanoscale/ }\end{array}$ \\
\hline Author contact & $\begin{array}{l}\text { your email steven.defeyter@kuleuven.be } \\
\text { your phone number }+32(0) 16327921\end{array}$ \\
\hline IR & https://lirias.kuleuven.be/handle/123456789/608818 \\
\hline
\end{tabular}




\title{
Hierarchical self-assembly of enantiopure and racemic helicenes at the liquid/solid interface: from 2D to 3D†
}

\author{
Hai Cao, $t^{\mathrm{a}}$ Andrea Minoia, $t^{\mathrm{b}}$ Inge De Cat, ${ }^{\mathrm{a}}$ Johannes Seibel, ${ }^{\mathrm{a}}$ Deepali Waghray, ${ }^{\mathrm{c}}$ \\ Zhi Li, ${ }^{a}$ David Cornil, ${ }^{b}$ Kunal S. Mali, (D) ${ }^{a}$ Roberto Lazzaroni, (D) ${ }^{b}$ Wim Dehaen (D) ${ }^{c}$ and \\ Steven De Feyter (D)*a
}

The performance of organic nanostructures is closely related to the organization of the functional molecules. Frequently, molecular chirality plays a central role in the way molecules assemble at the supramolecular level. Herein we report the hierarchical self-assembly of benzo-fused tetrathia[7]helicenes on solid surfaces, from a single surface-bound molecule to well-defined microstructures, using a combination of various characterization techniques assisted by molecular modeling simulations. Similarities as well as discrepancies are revealed between homochiral and heterochiral aggregations by monitoring the hierarchical nucleation of helicenes on surfaces, where the impact of enantiopurity, concentration and adsorbatesubstrate interaction on molecular organization are disclosed.

Received 8th September 2017 Accepted 2nd November 2017 DOI: $10.1039 / c 7 n r 06700 c$ rsc.li/nanoscale tion-based aggregations. ${ }^{15}$ Nevertheless, insight into the mechanism of transmission of chirality, from free monomers to bulk assemblies, remains elusive. There are many reasons to account for it. On the one hand, the acquisition of highly crystalline superstructures remains challenging, in particular for the racemate. On the other hand, conventional morphological and spectroscopic characterizations at macroscopic level usually cannot precisely describe the way molecules assemble. While a nanoscale imaging technique such as scanning tunneling microscopy (STM) can visualize two-dimensional networks on atomically flat surfaces, thereby bringing insight into transmission and amplification of chirality, ${ }^{16-18}$ most studies so far barely transcend monolayer coverage. It appears that no single technique can provide a complete picture of the propagation of chirality across multiple length scales.

Herein, we bridge the gap between molecular and macroscopic scale organization of helicenes by virtue of scrutinizing and comparing the hierarchical self-assembled structures obtained from the same solvent but on different solid surfaces. By using distinct methods to characterize the enantiopure and racemic molecular assemblies of helicenes on different substrates, we show that the intermolecular arrangements, down to the level of dimerization, are responsible for the complex crystalline forms at the supramolecular level. The variability in molecular organization is a manifestation of the many competing factors at interfaces, including the enantiopurity, concentration and substrate registry.

Helicenes constitute an interesting class of functional compounds ${ }^{19}$ that have been extensively investigated both in 
solution $^{7-11}$ and on solid surfaces. ${ }^{20-29}$ The molecular structures of the benzo-fused tetrathia[7]helicenes ${ }^{30}$ used in this study, termed as $(P)-1$ and $(M)-1$, are displayed in Fig. 1A. Each contains two pendent benzene rings and four lateral alkoxy substitutions. $(P)-1$ and (rac)- 1 (equimolar mixture of $(P)-1$ and $(M)$-1) were dissolved in 1,2,4-trichlorobenzene (TCB) and then deposited on different substrates (silicon, graphite and gold) for study. The solvent allows for concentration control and provides a medium for the diffusion of adsorbates, whereas the use of distinct substrates is intended to provide variable adsorbate-substrate interactions and facilitate different characterization methods. It has been suggested that strong interactions with the substrate ensure the immobilization of small aggregates that otherwise cannot be visualized due to their high mobility, while if the adsorbate interacts poorly with the substrate, the superstructure being formed is very much determined by the interaction between the adsorbates themselves. ${ }^{31,32}$ The strength of the adsorbate-substrate interaction was evaluated by calculating the adsorption energies of two typical compounds, coronene and hexadecane, on three substrates, and we found that these kinds of molecules interact very poorly with silicon, but strongly with gold (Table S1†).

Bulk self-assembled helicene structures were obtained on clean $\mathrm{Si}(100)$ wafers (covered by its native oxide) by drop casting and characterizing them by scanning electron microscopy (SEM). On the one hand, SEM images of $(P)-1$ (Fig. 1B and $\mathrm{S} 1 \dagger$ ) reveal extended dendritic nanostructures whose width ranges from $500 \mathrm{~nm}$ to $3 \mu \mathrm{m}$ and length reaches up to $100 \mu \mathrm{m}$. The crystallization of ( $\mathrm{rac})-1$, on the other hand, furnishes only seemingly amorphous nanostructures of no particular shape, as depicted in Fig. 1C and S2.† A non-racemic composition gives rise to sunflower-like nanoribbon clusters (Fig. S3†). The stark distinction in morphology is a clear indi-

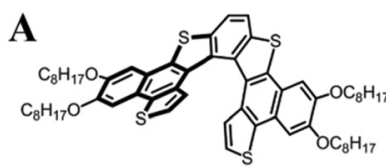

$(P)-1$

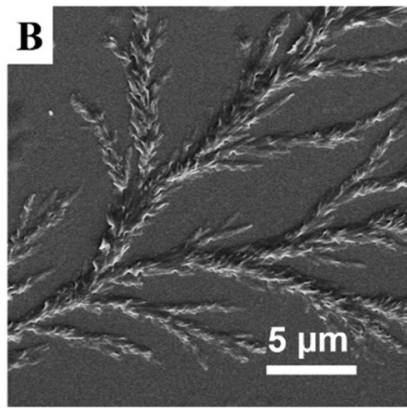

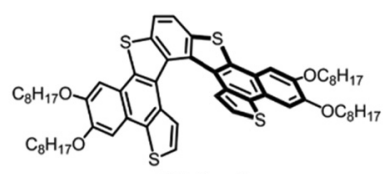

$(M)-1$

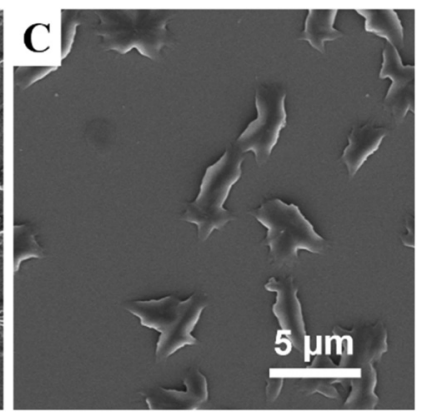

Fig. 1 (A) Chemical structures of the enantiomers of benzo-fused tetrathia[7]helicenes: $(P)-1$ and $(M)-1$. (B, C) SEM images of the nanostructures prepared via drop casting TCB $\left(C=5 \times 10^{-4} \mathrm{M}\right)$ solutions of $(P)-1$ and $(\mathrm{rac})-1$ on silicon surfaces, respectively. After the evaporation of the solvent, these samples were coated with gold for SEM characterization. cation that homo-/heterochiral recognitions are decisive driving forces that determine the molecular organization in bulk helicene self-assemblies, in line with previous studies demonstrating the correlation between morphology and enantiomeric excess. $^{3-9}$

Simply from the large scale characterization, however, it is not straightforward to tell how the enantiopurity affects macroscopic organization. To get insight into the molecular arrangement, surface-confined self-assembly was investigated using STM. There have been many studies on helicenes at the vacuum/metal interface,${ }^{20-26}$ but to a much lesser extent at the liquid/metal interface, ${ }^{29}$ not to mention on HOPG (highly ordered pyrolytic graphite) surface. Anyhow, we could not find any evidence for self-assembled monolayer formation by STM upon deposition of a TCB solution of $(P)-1$ on HOPG. A plausible reason for the lack of stable $2 \mathrm{D}$ networks may lie in the non-planar nature of $(P)-1$ and therewith poor affinity with the graphite surface, which is a common issue that has been often observed at the liquid/solid interface but can be improved by virtue of host-guest complementarity. ${ }^{29,33}$

In great contrast, $(\mathrm{rac})-1$ furnished well-organized bilayer networks under identical experimental conditions, as shown in Fig. 2A. This observation is rather unusual since the bilayer formation is observed even at submonolayer coverage (Fig. S4†). In addition, experiments carried out using 1-phenyloctane as solvent further confirm the propensity of ( $\mathrm{rac}$ ) -1 to form supramolecular bilayers (Fig. S5 $\dagger$ ). The network consists of dumbbell- domains extending over several thousand square nanometres, and we did not observe the existence of mirror

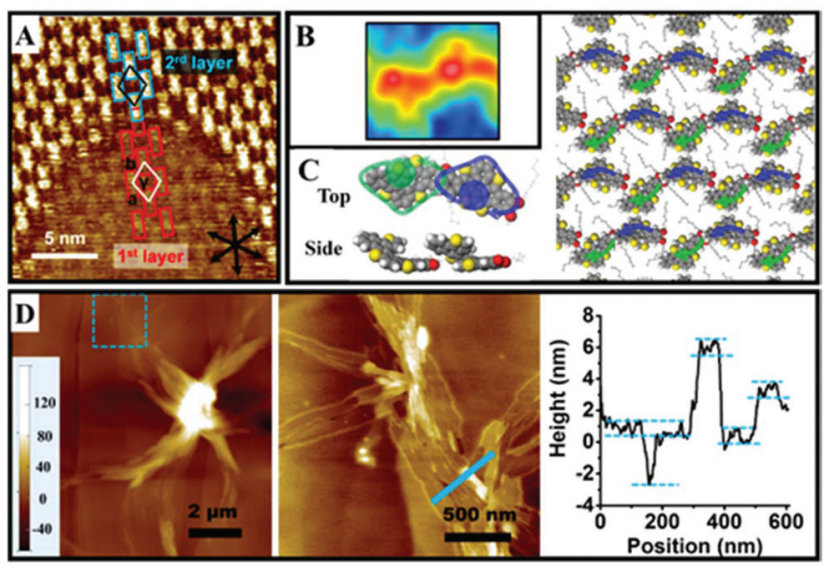

Fig. 2 (A) STM image of the bilayer network of (rac)-1 at the TCB/HOPG interface $\left(c=5 \times 10^{-4} \mathrm{M}\right)$. Red and light blue boxes map out the first and second layer, respectively. The black arrows indicate the main symmetry axes of underlying substrate. (B) Contrast image of a dumbbell feature. Note that the contrast feature of enantiopure $(P)-1$ on $\mathrm{Au}(111)$ (to be discussed in the following section) is used as a reference to assign the absolute chirality of each helicene (Fig. S6 $)$ ). (C) Top and side views of side-by-side complementary stacking of helicenes and calculated molecular arrangement on the basis of the bright spots observed in the STM images. $(P)-1$ and $(M)-1$ are differentiated with blue and green shapes. (D) AFM images of the surface morphology after solvent evaporation, and height profile analysis of a selected area. 
1 image domains shaped features arranged in a chessboard fashion, with domains extending over several thousand square nanometres, and we did not observe the existence of mirror image domains on the surface. The lower layer of helicene molecules can be seen through defects in the top layer, offering an important clue for the understanding of the structure of the ( $r a c)-1$ bilayer. Structurally, the two layers appear identical, and the bright dumbbells in these two layers have identical orientation, yet the lower layer is shifted with respect to the upper layer by one helicene molecule, as indicated by the red and light blue boxes in Fig. 2A. Close inspection of individual dumbbells reveals bright features that appear to be mirror images of each other (Fig. 2B and S6†). We attribute these features to the aromatic backbones of two helicene molecules, and therewith each dumbbell is thought to represent a racemic pair of helicene molecules.

Modelling was performed to further investigate the arrangement of the helicene molecules on graphite. An initial arrangement of heterochiral helicene pairs was inserted into a periodic modeling box having the same size as the experimentally obtained unit cell $\left(a=b=1.9 \pm 0.1 \mathrm{~nm}, \gamma=110 \pm 1^{\circ}\right)$ and oriented according to the STM images. In order to refine the structure of the monolayer, we applied an iterative Quench/MD scheme, consisting of alternating structure optimization and molecular dynamics simulations (see ESI† section for details). The resulting monolayer is in good agreement with that obtained experimentally (Fig. 2C). Close examination of the model monolayer structure reveals that there is shape complementarity between adjacent molecules: the part of the helicene core that is adsorbed on the surface in one molecule gets inserted beneath the raised part of the core in the adjacent molecule. Such lateral complementarity exists between alternatively arranged $(P)-1$ and $(M)-1$ molecules, but cannot apply to a continuous homochiral monolayer where only one enantiomer is present, implying that the heterochiral interaction is the plausible driving force that stabilizes the bilayer networks on the graphite surface.

Considering the alternating arrangement of $(P)-1$ and $(M)-1$, a shift of one helicene molecule between two layers suggests that the helicity of the overlapped molecules in these two layers is opposite, therefore complementary heterochiral $\pi-\pi$ stacking along the helix axis accounts for the formation of bilayer networks. Such a heterochiral arrangement in both layers is distinct from that observed by Ernst et al. ${ }^{25,26}$ where multilayers are piled up by homochiral monolayers of alternating chirality. A possible reason for such difference may lie in the fact that the adsorption of helicenes on the graphite surface is much weaker than that on $\mathrm{Cu}(111)$ : in our case, the bilayer formation is to a large extent determined by intermolecular interactions between the adsorbates themselves, as evidenced by the observation of bilayer structures at submonolayer coverage, whereas the transition from a $2 \mathrm{D}$ to a $3 \mathrm{D}$ racemate on $\mathrm{Cu}(111)$ only occurs when the deposit exceeds full monolayer coverage.

There is no experimental evidence provided by STM for the formation of multilayers beyond bilayers. We further employed atomic force microscopy (AFM) to probe the surface morphology after the evaporation of solvent, as displayed in Fig. 2D. Irregular bright protrusions were observed, alike that on silicon surface. Close inspection reveals flake-like structures inthe vicinity, and the seemingly amorphous protrusions appear to be islands made of lots of layers. Height profile analyses disclose that the thickness of each layer is not random but amounts to $\sim 1 \mathrm{~nm}, \sim 2 \mathrm{~nm}$ or $\sim 3 \mathrm{~nm}$, i.e., approximately the height of two layers, four layers and six layers of helicene networks, respectively (Fig. 2D and S7 $\dagger$ ). The aggregation of (rac)-1, on both silicon and graphite surfaces, is therefore a manifestation of the Stranski-Krastanov mode, namely, the 'layer-plus-island growth' (Scheme 1).

We then moved to the investigation of the self-assembly on gold surface, on which strong adsorption is expected to limit the diffusion of helicenes. The gold-sulfur interaction is a key player in the field of self-assembled monolayers (SAMs) and it may play a role in the interaction of these helicenes with the gold surface. It must be noted that the nature of the $\mathrm{S}-\mathrm{Au}$ interaction can be quite diverse. ${ }^{34}$ On one hand, a strong covalent bond is observed for SAMs based on thiol-containing molecules, such as alkanethiols. ${ }^{35}$ On the other hand, in the case of sulfur-containing heteroaromatic compounds such as thiophene, quantum-chemical calculations predict no significant bonding between the sulfur and gold atoms and the molecules tend to adsorb parallel to the surface plane, rather than in a perpendicular configuration. ${ }^{35}$ To illustrate how the nucleation and growth of helicenes occur and how homo-/ heterochiral interactions affect self-assembled network formation, we studied the stepwise aggregation of helicenes at the TCB/Au (111) interface. Indeed, more information on sub-monolayer assemblies was gleaned, as depicted in Fig. 3 . The variation of the concentration in solution phase, from $2 \times 10^{-6} \mathrm{M}$ to $5 \times$ $10^{-4} \mathrm{M}$, has a significant impact on the way helicenes arrange for both the enantiopure and racemic samples. Fig. 3A-D and Fig. S8 $\uparrow$ show the transition of $(P)-1$, from a single surfacebound monomer at the elbow site of the herringbone reconstruction to bilayers. The head-to-head adsorbed dimers that show up at the elbows (Fig. 3A) represent the optimum arrangement of two molecules of the same chirality, but the continuous head-to-tail linear alignment appears to be more favorable for further growth. Rows of $(P)-1$ (Fig. 3B, C and J) gradually populate the surface, along with the disappearance of small clusters in between. When the deposit exceeds full surface coverage, a second layer forms on top, with identical orientation or rotated by $60^{\circ}$ with respect to the first layer (Fig. 3D and S9†).

To gain a better understanding of the influence of heterochiral recognition on surface pattern transformation, nonequimolar mixtures of $(P)-1$ and $(M)-1$ on $\mathrm{Au}(111)$ were investigated. Surprisingly, we found that $5 \%$ of $(M)-1$ in the mixture was enough to induce significant changes in the $(P)-1$ monolayer (Fig. $4 \mathrm{~A}$ and $\mathrm{S} 15 \dagger$ ). Although a majority of the surface is still covered with ordered columns that are characteristic of the $(P)-1$ assembly, there are a number of smaller aggregates with no specific orientation in between the ordered columns. 

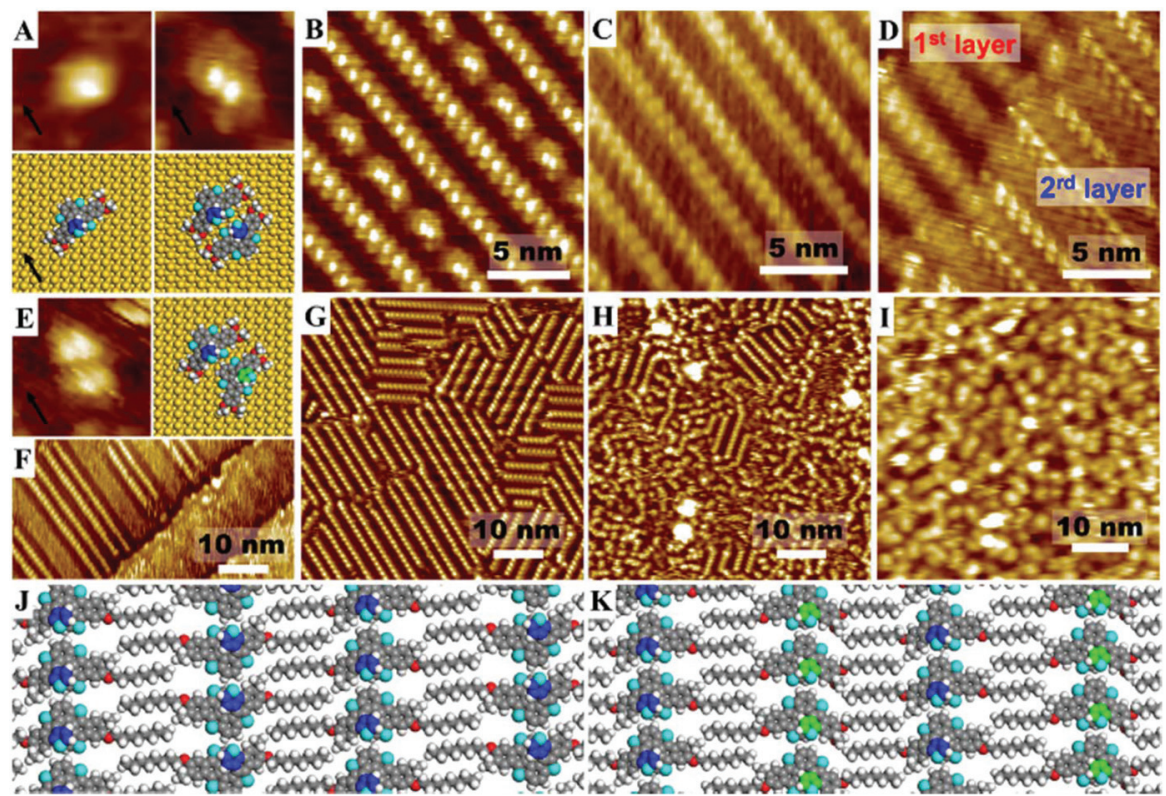

Fig. 3 (A-D) Evolution of the self-assembled structures of $(P)-1$ at the TCB/Au(111) interface with increasing concentration. Concentration: $A, 2 \times$ $10^{-6} \mathrm{M} ; \mathrm{B}, 1 \times 10^{-5} \mathrm{M} ; \mathrm{C}$ and $\mathrm{D}, 5 \times 10^{-4} \mathrm{M}$. Size: $\mathrm{A}, 3 \times 3 \mathrm{~nm}^{2}$. (E-l) Concentration-driven order-to-disorder transition in the self-assembled networks of (rac)- 1 at the TCB/Au(111) interface. Concentration: $E$ and F, $5 \times 10^{-6} \mathrm{M} ; \mathrm{G}, 1 \times 10^{-5} \mathrm{M} ; \mathrm{H}, 5 \times 10^{-5} \mathrm{M}, \mathrm{I}, 5 \times 10^{-4} \mathrm{M}$. Size: E, $3 \times 3 \mathrm{~nm}$. The models of monomer and dimer of helicenes are calculated using force field methods. Details see Fig. S11-S13. $\dagger$ Black arrows indicate the $<11 \overline{2}>$ orientation of the substrate. $(\mathrm{J}, \mathrm{K})$ Tentative structural models for the linear networks of $(P)-1$ and $(\mathrm{rac})-1$ on the basis of close-up STM observations depicted in Fig. S14. $\dagger$ The protruding thiophene groups of $(P)-1$ and $(M)-1$ are highlighted in blue and green, respectively. Only two alkyl chains of a helicene are displayed while considering the spacing between helicenes. The seemingly alternating inter-row spacing is presumably a consequence of the different orientation, or helicity, of the helicenes in adjacent rows.
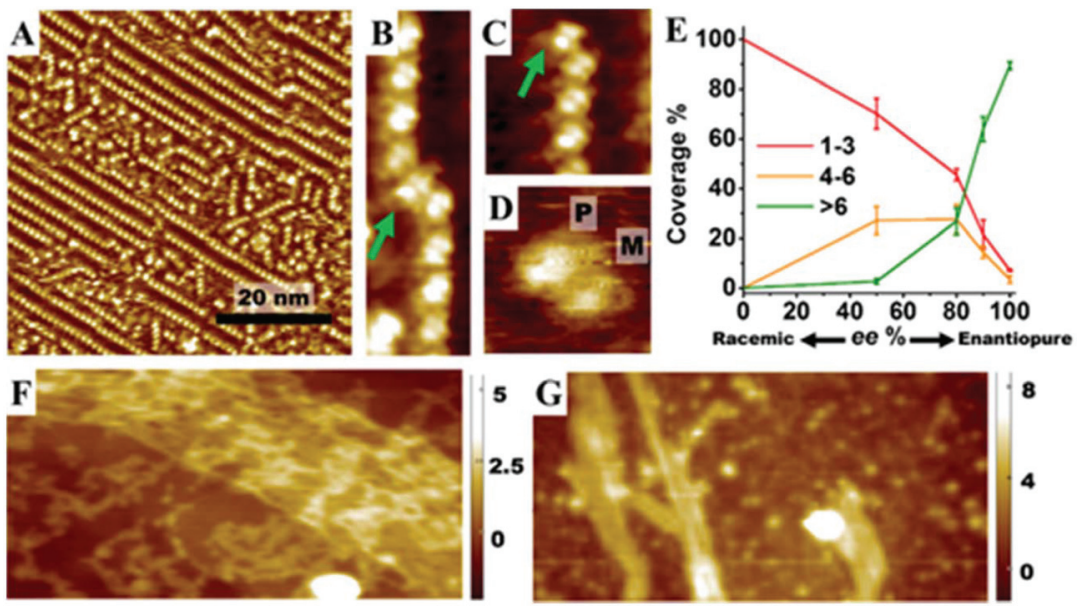

Fig. 4 (A) Large-scale STM image of the self-assembled network of $(P)-1:(M)-1=95: 5$ at the TCB/Au(111) interface $\left(c=5 \times 10^{-4} M\right)$. (B-D) Frequently observed defects in the columnar stacking when mixing 5\% (M)-1 and 95\% (P)-1: (B) misalignment and (C) break of helicene rows, and (D) heterodimer of $(P)-1$ and $(M)-1$. Helicenes appear with an orientation different to the vast majority are indicated with green arrows. (E) Surface coverage of the aggregates with different lengths (number of molecules in each column) as a function of ee. ee $=([(P)-1]-[(M)-1]) /([(P)-1]+[(M)-1]) \times$ $100 \%$. A kink is treated as the end of a column. $(F, G)$ AFM images $\left(0.5 \times 1 \mu^{2}\right)$ of the surface morphology of $(P)-1$ and $(r a c)-1$, respectively, on $\mathrm{Au}(111)$ after the evaporation of solvent.

Inspection of STM images revealed that frequently a column of $(P)-1$ bends or breaks (Fig. 4B and C), with the concomitant appearance of small clusters (Fig. 4D). At such sites, a helicene molecule appears with an orientation different from the others, and the spacing between the molecules with different features is shorter than that within a $(P)-1$ column. We tentatively attribute such features to $(M)-1$ molecules. The concentration-driven order-to-disorder transition of ( $\mathrm{rac}$-1 1 is therefore a consequence of $2 \mathrm{D}$ racemate to $1 \mathrm{D}$ racemate conversion in pursuit of higher packing density. The degree of disorder 
becomes significant when the content of $(M)-1$ is further increased (Fig. S16 $\dagger$ ). A detailed analysis of the degree of organization in response to a decrease in enantiomeric excess is given in Fig. 4E, showing dramatic reduction of long aggregates with a concomitant increase in the presence of smaller aggregates.

The concentration-driven aggregation of $(\mathrm{rac})-1$ is somewhat different (Fig. 3E-I and S10†). Three steps can be identified. First, two helicene molecules of opposite chirality dimerize through a head-to-tail complementary arrangement (Fig. 3E), different from that on graphite. Further adsorption of helicenes immediately leads to the transition into linear rows. Interestingly, the number of helicene rows in a given domain is always even (Fig. 3F, G). A model for such a linear network is given in Fig. 3K, showing a $1 \mathrm{D}$ homochiral but $2 \mathrm{D}$ racemic organization. Upon increasing the concentration, ( $\mathrm{rac}$ )-1 generates full monolayer coverage of linear networks. At this point the surface structures of $(P)-1$ and (rac)-1 are very similar. Nevertheless, a further increase of the concentration of $(\mathrm{rac})-1$ leads to the emergence of disordered areas, instead of the formation of a bilayer (Fig. $3 \mathrm{H}$ ). At the concentration of $5 \times 10^{-4}$ M, no ordered assembly can be identified anymore (Fig. 3I). From the disordered area shown in the STM images, however, it is hard to identify the factor(s) that leads to the order-to-disorder transition, in particular considering that the same solution leads to well-defined networks on graphite.

The reason why ( $\mathrm{rac}$ )-1 furnishes distinct organizations on two different surfaces can be understood by a slight difference in the manner of complementary stacking between two helicenes of opposite chirality. On HOPG, the formation of extended alternating arrangements relies on side-by-side stacking of the helicenes. In contrast, the heterochiral dimer adsorbed on $\mathrm{Au}(111)$ adopts a head-to-tail arrangement, which is presumably unfavorable for further stacking into extended networks. The difference in arrangement, we believe, is a manifestation of distinct adsorbate-substrate interactions at interfaces.

The linear networks of $(P)-1$ and randomly adsorbed clusters of ( $\mathrm{rac}$ )-1 propagate to larger scales as nanofibers (Fig. $4 \mathrm{~F}$ and $\mathrm{S} 17 \dagger$ ) and amorphous aggregates (Fig. $4 \mathrm{G}$ and $\mathrm{S} 18 \dagger$ ), respectively. Surprisingly, the same flake-like microstructures of $(r a c)-1$ were observed on top of the amorphous layer, possibly due to the decreased influence of the substrate on the adsorbate organization at a certain distance.

Note that the side-by-side zigzag array in a racemic latti$c e,^{20,21 b}$ the head-to-head configuration of a homochiral dimer, ${ }^{26}$ the head-to-tail arrangement of a heterochiral dimer $^{24}$ and 1D homochiral rows ${ }^{21}$ have already been revealed in different helicene systems, yet these variable spatial arrangements on surfaces have never been associated with structural diversity at the macroscopic level. In our approach, we observed the variability of intermolecular on-surface arrangements in a way that the molecular organization supports the constantly changing needs for the strongest mutual interactions, optimum adsorption and growth and higher packing density. ${ }^{37}$ The many ways helicenes assemble even- tually evolve into different supramolecular and macroscopic structures.

In summary, we have explored the hierarchical self-assembly of enantiopure and racemic helicenes at the liquid/solid interface, from small clusters to well defined microstructures. The distinction in morphology in the bulk phase, going from enantiopure to racemic self-assembly, can be explained by molecular level information obtained from STM measurements carried out on self-assembled monolayers, supported by molecular modeling simulations. Despite certain limitations, this study may provide an alternative approach to understand the structural diversity of chiral self-assembly. Additionally, such an integrated approach may serve as a basis for the modulation of self-assembled structures for the engineering of functional supramolecular materials.

\section{Conflicts of interest}

वा1

\section{Acknowledgements}

We thank Dr Liang Su and Dr Haifeng Yuan (KU Leuven) for the SEM measurements. This work is supported by the Fund of Scientific Research Flanders (FWO), KU Leuven (GOA 11/ 003). The Leuven/Mons collaboration is supported by the Science Policy Office of the Belgian Federal Government (IAP 7/5). Research in Mons is also supported by the OPTI2MAT Excellence Program of Région Wallonne and FNRS-FRFC (CECI HPC consortium). The research leading to these results has also received funding from the European Research Council under the European Union's Seventh Framework Programme (FP7/2007-2013)/ERC Grant Agreement No. 340324 to S. D. F. H. C. is an FWO Pegasus Marie Curie Fellow.

\section{Notes and references}

1 N. Katsonis, E. Lacaze and B. L. Feringa, J. Mater. Chem., 2008, 18, 2065-2073.

2 Y. Wang, J. Xu, Y. W. Wang and H. Y. Chen, Chem. Soc. Rev., 2013, 42, 2930-2962.

3 M. H. Liu, L. Zhang and T. Y. Wang, Chem. Rev., 2015, 115, 7304-7397.

4 C. P. Brock, W. B. Schweizer and J. D. Dunitz, J. Am. Chem. Soc., 1991, 113, 9811-9820.

5 R. Oda, I. Huc, M. Schmutz, S. J. Candau and F. C. MacKintosh, Nature, 1999, 399, 566-569.

6 X. F. Zhu, Y. G. Li, P. F. Duan and M. H. Liu, Chem. - Eur. J., 2010, 16, 8034-8040.

7 T. Verbiest, S. Van Elshocht, M. Kauranen, L. Hellemans, J. Snauwaert, C. Nuckolls, T. J. Katz and A. Persoons, Science, 1998, 282, 913-915.

8 T. Kaseyama, S. Furumi, X. Zhang, K. Tanaka and M. Takeuchi, Angew. Chem., Int. Ed., 2011, 50, 3684-3687. 
9 J. Kumar, H. Tsumatori, J. Yuasa, T. Kawai and T. Nakashima, Angew. Chem., Int. Ed., 2015, 54, 5943-5947.

10 C. Nuckolls and T. J. Katz, J. Am. Chem. Soc., 1998, 120, 9541-9544.

11 K. E. S. Phillips, T. J. Katz, S. Jockusch, A. J. Lovinger and N. J. Turro, J. Am. Chem. Soc., 2001, 123, 11899-11907.

12 F. Pop, P. Auban-Senzier, A. Frąckowiak, K. Ptaszyński, I. Olejniczak, J. D. Wallis, E. Canadell and N. Avarvari, J. Am. Chem. Soc., 2013, 135, 17176-17186.

13 F. Pop, P. Auban-Senzier, E. Canadell and N. Avarvari, Chem. Commun., 2016, 52, 12438-12441.

14 W. J. Zou, Y. Yan, J. Fang, Y. Yang, J. Liang, K. Deng, J. L. Yao and Z. X. Wei, J. Am. Chem. Soc., 2014, 136, 578581.

15 (a) L. Pérez-Garcíaa and D. B. Amabilino, Chem. Soc. Rev., 2002, 31, 342-356; (b) L. Pérez-García and D. B. Amabilino, Chem. Soc. Rev., 2007, 36, 941-967.

16 J. A. A. W. Elemans, I. De Cat, H. Xu and S. De Feyter, Chem. Soc. Rev., 2009, 38, 722-736.

17 (a) R. Raval, Chem. Soc. Rev., 2009, 38, 707-721; (b) S. Haq, N. Liu, V. Humblot, A. P. J. Jansen and R. Raval, Nat. Chem., 2009, 1, 409-414.

18 K. H. Ernst, Phys. Status Solidi B, 2012, 249, 2057-2088.

19 Y. Shen and C. F. Chen, Chem. Rev., 2012, 112, 1463-1535.

20 (a) R. Fasel, M. Parschau and K. H. Ernst, Nature, 2006, 439, 449-452; (b) J. Seibel, M. Parschau and K. H. Ernst, J. Phys. Chem. C, 2014, 118, 29135-29141.

21 (a) M. Stöhr, S. Boz, M. Schr, M. T. Nguyen, C. A. Pignedoli, D. Passerone, W. B. Schweizer, C. Thilgen, T. A. Jung and F. Diederich, Angew. Chem., Int. Ed., 2011, 50, 9982-9986; (b) A. Shchyrba, M. T. Nguyen, C. Wäckerlin, S. Martens, S. Nowakowska, T. Ivas, J. Roose, T. Nijs, S. Boz, M. Schär, M. Stöhr, C. A. Pignedoli, C. Thilgen, F. Diederich, D. Passerone and T. A. Jung, J. Am. Chem. Soc., 2013, 135, 15270-15273.

22 (a) M. Taniguchi, H. Nakagawa, A. Yamagishi and K. Yamada, Surf. Sci., 2000, 454-456, 1005-1009; (b) M. Taniguchi, H. Nakagawa, A. Yamagishi and K. Yamada, Surf. Sci., 2002, 507-510, 458-462; (c) M. Taniguchi, H. Nakagawa, A. Yamagishi and 1 K. Yamada, J. Mol. Catal. A: Chem., 2003, 199, 65-71. Q5

23 K. H. Ernst, Acc. Chem. Res., 2016, 49, 1182-1190.

24 K. H. Ernst, S. Baumann, C. P. Lutz, J. Seibel, L. Zoppi and A. J. Heinrich, Nano Lett., 2015, 15, 5388-5392.

25 M. Parschau and K. H. Ernst, Angew. Chem., Int. Ed., 2015, 54, 14422-14426.

26 (a) A. Mairena, L. Zoppi, J. Seibel, A. F. Tröster, K. Grenader, M. Parschau, A. Terfort and K. H. Ernst, ACS Nano, 2017, 11, 865-871; (b) J. Seibel, O. Allemann, J. S. Siegel and K. H. Ernst, J. Am. Chem. Soc., 2013, 135, 7434-7437.

27 C. Hauke, P. Rahe, M. Nimmrich, J. Schütte, M. Kittelmann, I. Stará, I. Stary, J. Rybácek and A. Kühnle, J. Phys. Chem. C, 2012, 116, 4637-4641.

28 P. Rahe, M. Nimmrich, A. Greuling, J. Schütte, I. G. Stará, J. Rybáček, G. Huerta-Angeles, I. Starý, M. Rohlfing and A. Kühnle, J. Phys. Chem. C, 2010, 114, 1547-1552.

29 T. Balandina, M. W. van der Meijden, O. Ivasenko, D. Cornil, J. Cornil, R. Lazzaroni, R. M. Kellogg and S. De Feyter, Chem. Commun., 2013, 49, 2207-2209.

30 D. Waghray, W. Nulens and W. Dehaen, Org. Lett., 2011, 13, 5516-5519.

31 D. B. Amabilino, in Supramolecular Chemistry at Surfaces, RSC Publishing, Cambridge, 2016, pp. 340-341.

32 V. Palermo and P. Samorì, Angew. Chem., Int. Ed., 2007, 46, 4428-4432.

33 O. Ivasenko, J. M. MacLeod, K. Yu Chernichenko, E. S. Balenkova, R. V. Shpanchenko, V. G. Nenajdenko, F. Rosei and D. F. Perepichka, Chem. Commun., 2009, 1192-1194.

34 E. Pensa, E. Cortés, G. Corthey, P. Carro, C. Vericat, M. H. Fonticelli, G. Benítez, A. A. Rubert and R. C. Salvarezza, Acc. Chem. Res., 2012, 45, 1183-1192.

35 J. C. Love, L. A. Estroff, J. K. Kriebel, R. G. Nuzzo and G. M. Whitesides, Chem. Rev., 2005, 105, 1103-1170.

36 L. Buimaga-Iarinca and C. Morari, Theor. Chem. Acc., 2014, 133, 1502-1512.

37 C. P. Brock, W. B. Schweizer and J. D. Dunitz, J. Am. Chem. Soc., 1991, 113, 9811-9820. 